\title{
COMPARISON BETWEEN OMENTOPLASTY AND PARTIAL CYSTECTOMY AND DRAINAGE (PCD) TECHENQUES IN SURGICAL MANAGEMENT OF HYDATID CYSTS LIVER IN ENDEMIC AREA (YEMEN) \\ By \\ MARWAN MANSOUR BORHAM \\ Department of Surgery, Faculty of Medicine, Al-Azhar University, Nasr City, Egypt
}

\begin{abstract}
Hydatisosis caused by Echinococcus granulosus is more or less endemic in all sheep raising countries. This study evaluated omentoplasty versus partial cystectomy with drainage in surgical management of hydatid cysts of the liver in endemic area (Yemen). A total of 60 patients with hydatid cyst in the liver was divided into two groups; G1 (32 patients) treated with omentoplasty and G2 (28patients) treated with partial cystectomy with drainage (PCD).

The results showed that the wound sepsis was seen in $6.25 \%$, of G1and in $14.2 \%$ of G2, biliary leakage occurred in $3.5 \%$ of G2, intra-abdominal abscess formation occurred in $3,5 \%$ of G2, atelectasis was in $3.1 \%$ of G1 and in $3.5 \%$ of G2. The total morbidity was $12.5 \%$ in G1 and $32.1 \%$ in G2. There was a significant difference between the two groups as regard to the mean post-operative hospital stay, (6.5 days in G1 versus 15.6 days in G2).
\end{abstract}

Key words: Yemen, Hydatid cysts, Omentoplasty, Partial cystectomy, Surgical drainage

\section{Introduction}

Ecchinococcal (hydatid) disease has been known since the time of Hippocrates, who describes it as liver full of water (Kassem et al, 2013). The disease is zoonosis man and other herbivorous animals are infected by ingestion of its eggs dropped from the definitive host; dogs (Hussein et al, 2012). Hydatidosis the other neglected tropical diseases (NTDs) are highly endemic but patchily distributed among the 20 countries and almost 400 million people of the Middle East and North Africa (MENA) region, and disproportionately affect an estimated 65 million people living on less than US\$2 per day (Hotez et al, 2012). Human cystic echinococcosis, with hepatic localization as its most common clinical manifestation (50 to $75 \%$ ) of about $72 \%$ in the right lobe, the range of cysts varied from $1 \mathrm{~cm}$ to $30 \mathrm{~cm}$ (Just et al, 2014). Most of hydatid is asymptomatic and most of symptomatized are complicated, the complications are present in one third of cases, with life threatening inetrabiliary rupture unless dealt with properly (Ozkan et al, 2013). The diagnosis of hydatid cyst is based on laboratory, immunological and radiological tests as well as ultrasound, which is the most useful noninvasive diagnostic test, and classified the cyst (Kizildag et al, 2013). Nevertheless, computed tomography provides better information regarding the location and size of the cyst (Yucesoy et al, 2013). In hydatidosis the basic treatment is surgery. Main objective of the surgery should be parenchyma sparing while taking off completely the cysts. Although the disease is frequently seen in liver and lung, other organ involvements should be considered and no problem where hydatid cyst in abdomen and thorax should be attentively controlled by the simplest imaging method also outside of clinical symptoms (Eser et al, 2013). Surgical treatment varies from complete resection (e.g. peri cystectomy \& hepatectomy), to minimal invasive procedures (e.g. percutaneous aspiration of cyst), but the ideal treatment is still controversial (Sahin et al, 2010). The choice of therapy depends on several factors; number, localization of the cysts, surgeon experience, and presence of complications (Trai- 
bi et al, 2012). Unfortunately, multiplicity of cyst is still common surgical problem, sometimes management is difficult, requiring operative experience in this filed (Tan et al, 1998).

This worK studied the management and outcome of patients with liver hydatid cysts in Yemen.

\section{Patients, Materials and Methods}

This is prospective comparative study cover the period from March 2009 to March 2011, on 60 patients with further two years follow up, in the department of surgery at Al-Salam Hospital, at Saada Republic Yemen. The patients were divided into two groups; G1 consisted of 32 patients treated with omentoplasty and G2 28 patients treated with partial cystectomy with drainage of the cyst (PCD), which means drainage of the cyst contents, partial excision of the wall and running suture of the edge with chromic sutures. In G1, 20 cases $(62.5) \%$ were females and 12 cases $(37.5) \%$ were male.in G2 ,18 cases $(64.3 \%)$ were females and 10 cases $(35.7 \%)$ were males. The mean age of the patients in G1 was 30 \pm 14 (ranged 11-50 years) in G2 was 37.3 \pm 13.5 (ranged 13-53 years). Surgical techniques were compared with post-operative complications, hospital stay and recurrence of the disease.

Preoperative evaluation: All patients are subjected to detailed history, physical examination, and all routine investigations such as, complete blood picture, liver function tests, kidney function tests, coagulation profile, ECG, chest x-ray and ultrasonography which was the main tool in diagnosis of hydatid cyst. Computed tomography was indicated in which the result of ultrasound was not conclusive or equivocal. The most important indication of computed tomography was anatomical cystic details, multiple cyst solid components inside the cyst. If there was suspicion of infection preoperative broad spectrum antibiotics received. Inclu-

In the post-operative period, all patients were treated by albendazol therapy for three courses, each of 4 weeks with a gape of one sions criteria include, patient with single hydatid cyst of the liver, adult patient, noncomplicated hydatid cysts and patients with single surgical procedures. Exclusion criteria included all patients with extra hepatic cyst as lung, kidney or other organ hydatid cysts, multiple cysts of the liver, patient with other comorbidity, which may lengthen the hospital stay, patient with small, localized hydatid cysts liver which candidate for total excision of the cyst or hepatectomy, and complicated hydatid cyst of the liver.

Surgical technique: After the diagnosis, patients were prepared for operation and surgical intervention. After scrubbing extended right subcostal incision done, the area around the cyst was covered with packs soaked with 0.5 cetremid saline to prevent the further spread of the parasite during evacuation of the cyst. Cyst contents were aspirated from a suitable surface with a needle. After aspiration the cyst was injected with 0.5 cetrimide saline to sterilize the contents of the cyst, then $1-2 \mathrm{~cm}$ incision at the site of the needle to evacuate the contents of the cysts. The germnative membrane and daughter cysts were removed with forceps or spoons. With removal of redundant part of cysts excellent exposure was obtained. After evacuation of the cyst, the cyst cavity was managed by irrigation with 0.5 cetrimide saline for 10 to 15 minutes. The cavity is filled with worm saline, inspected for bile leakage and packed with dry, white packs for examination for bile leakage. Communications was managed by non-absorbable suture. Then the cyst cavity was filled with cetrimide saline immersed sponges for few minutes to sterilize the cavity of the cyst, and then sponges removed. At this point, the first group managed by filling the cavity with omentum (Fig. 1) and second group were treated with partial excision of cyst wall and suturing of the edge by absorbable chromic suture (Fig. 2).

week. All patients were followed up 2 times per month for the first three months, then 
every month for one year and then every three months for another one year.

\section{Results}

The hydatid cyst was in the right lobe of the liver in 39 cases $(65 \%)$ of total number of the patients, 22cases (68.75\%) in G1and 17 cases $(53.2 \%)$ in G2. Hydatid cysts were in the left lobe in 21 cases of total cases. About $64 \%$ liver lived in the rural areas and $84 \%$ are animal owner (sheep, dog and/or cat). This indicated that disease was common in rural areas than urban ones, were domestic and stray animals were common (Haridy et al, 2008). There were no significant differences regarding to the mean age, sex, location of hydatid cyst in the liver, residences of patients and animal owner between groups (Tab. 1).

This study the hydatidosis complications were wound sepsis, biliary leakage, intraabdominal abscess, bleeding, recurrence and atelectasis. Wound sepsis was in 2 patients of $\mathrm{G} 1(6.25 \%)$ and in 4 patients in G2(14.2\% ), biliary leakage was not in G1 only 1 case in G2 $(3.5 \%)$, intra-abdominal abscess formation was only in 1 case of G2 $(3,5 \%)$, atelectasis was in 1 case of $\mathrm{G} 1$ $(3.1 \%)$ and 1 case of G2 (3.5\%), the total morbidity were 4 case in G1 $(12.5 \%)$ and 9 cases in G2 (32.1\%) (Tab. 2)

The mean post-operative hospital stay was 6.5 days in $\mathrm{G} 1$ and 15.6 days in $\mathrm{G} 2$, with a significant difference between both groups.

Table 1: Characters of patients in both groups

\begin{tabular}{|l|c|c|c|}
\hline Characters of both groups & Omentoplasty G1(N=32) & PCD G2 $(\mathrm{N}=28)$ & P-value \\
\hline Age $($ Mean \pm SD) & $30.2 \pm 14$ & $27.3 \pm 13.5$ & 0.4190 \\
\hline -Female $(\mathrm{n}=38) 63.3 \%$ & $(\mathrm{n}=20) 62.5 \%$ & $(\mathrm{n}=18) 64.3 \%$ & 0.9012 \\
- Male $(\mathrm{n}=22) 36.7 \%$ & $(\mathrm{n}=12) 37.5 \%$ & $(\mathrm{n}=10) 35.7 \%$ & 0.9011 \\
\hline Location of cyst in liver & & & \\
- Right lobe $(\mathrm{n}=39) 65 \%$ & $(\mathrm{n}=22) 68.75 \%$ & $(\mathrm{n}=17) 53.2 \%$ & 0.3325 \\
- Left lobe $(\mathrm{n}=21) 35 \%$ & $(\mathrm{n}=11) 34.8 \%$ & & \\
\hline Patients' residence & $(\mathrm{n}=10) 35.7 \%$ & 0.8429 \\
- Rural area $(\mathrm{n}=38) 63.3 \%$ & $(\mathrm{n}=12) 62.5 \%$ & $(\mathrm{n}=18) 64.3 \%$ & 0.9012 \\
- Urban area $(\mathrm{n}=22) 36.7 \%$ & $(\mathrm{n}=10) 35.7 \%$ & 0.9011 \\
\hline Pet animal $(\mathrm{n}=50) 83.3 \%$ & $(\mathrm{n}=27) 84.4 \%$ & $(\mathrm{n}=23) 82.2 \%$ & 0.9051 \\
\hline
\end{tabular}

Table 2: Postoperative complication of both groups (omentoplasty and PCD)

\begin{tabular}{|l|c|c|c|}
\hline Complication & Omentoplasty G1(N=32) & PCD G2(N=28) & p-value \\
\hline Wound sepsis & $2(6.25 \%)$ & $4(14.2 \%)$ & 0.5526 \\
\hline Biliary leakage & $0 \%$ & $1(3.5 \%)$ & 0.9631 \\
\hline Intera abdominal abscess & $0 \%$ & $1(3.5 \%)$ & 0.9631 \\
\hline Bleeding & $1(3.1) \%$ & $2(7.1 \%)$ & 0.9077 \\
\hline Atelectasis & $1(3.1 \%)$ & $1(3.5 \%)$ & 0.5228 \\
\hline Total morbidity & $4(12.5) \%$ & $9(32.1 \%)$ & 0.1273 \\
\hline Hospital stay (mean hours) & $155 \pm 46$ & $374.4 \pm 119.6$ & 0.0000 \\
\hline
\end{tabular}

\section{Discussion}

Hydatid disease is worldwide health problem. The most common sites of hydatid cysts are the liver as its route of infection is portal circulation through absorption by intestinal mucosa, although more prevalent in sheep raising countries (Ghartimagar et al, 2013). Sites other than liver were lung, urinary tract and almost all body organs (ElSebaie et al, 2006). Hepatic hydatid cysts are single in common but in endemic areas, a big number of cases are multiple, but still common in the right lobe of the liver. Asymptomatic cysts may persist for long time years without complaints from the patient and may be discovered accidentally on examination. Hepatic hydatid cysts still a big problem in countries of endemic areas. Considerable number of hydatosis patient is dog, 
cats' owner, so prevention of hydatosis is possible by following fundamental hygienic measures. In hydatid cysts of the liver most of cases are asymptomatic 38 to $60 \%$ of all cases (Khuroo et al, 1997), but in the present study all cases were manifested and common symptom was pain in the right upper hypochonderium with physical finding was right hypochonderial swelling. Hydatid cysts should be suspected in endemic areas as there are no specific symptoms or signs (Ramia et al, 2013).

Ultrasonography is the most sensitive diagnostic tools and also used to classify the hydatid cyst with specificity and sensitivity up to $98 \%$ respectively (Hołody-Zaręba et al, 2013). In the present study, the accuracy of diagnosis depends on ultrasonography examinations, confirmed at surgery, with specific indications for CAT scan as anatomical cystic details, relation to other important structures, obstructive jaundice and solid component. The CT and M.R.I. are the method of choice (Grossi et al, 1991). Surgical treatment is still choice in the management of hydatid disease; the main goal of surgical treatment is to eradicate the parasite, to prevent intraoperative spillage of cyst contents and to obliterate the residual cavity surgical procedures have been advocated to achieve these goals (Doty and Tompkins, 1989). Scoleces in the cyst have been first inactivated with scolicidal agents in the present study cetremid saline $0.5 \%$ was used. Applied into the cystic cavity and 15 minutes should be allowed to pass. One should avoid use of this agent if there was a communication between the cyst and biliary tract because of the risk of sclerosing cholangitis. The two main surgical approaches in our study are omentoplasty obliteration of the cyst cavity after evacuation of the cystic contents without drainage and partial cystectomy with drainage (PCD) technique. Hepatic resections should be performed for the cysts of relatively small size and sub capsular location whereas anatomic resections should be performed for the cysts impairing most of the hepatic segments (Abbas et al, 2006). Drainage procedures can be followed by surgical complications post-operative, such as hepatic abscess, biliary fistulas, and long hospital stay, therefore omentoplasty for single or hydatid cysts of the liver causes less complications and shorter hospital stays than capsullarphy and drainage procedures (Fan et al, 2013). In other study including omentoplasty for multiple hepatic cysts showed the good result as our study. The postoperative complication rate of hepatic hydatid cyst has been reported to range from 6 to $47 \%$ (Palanivelu et al, 2007). In the present study, there was no mortality during or after operation and post-operative complication rate was $12.5 \%$ in $\mathrm{G} 1$ and $32.1 \%$ in $\mathrm{G} 2$. In patients treated by partial cystectomy with drainage (PCD) increased postoperative morbidity in cases of hepatic cyst in comparison to omentoplasty and the differences was significant, while partial cystectomy and drainage in the majority of cases, retain simple and safe procedures (Safioleas et al, 2006). Omentoplasty seems to be the best possible surgical alternative for the radical treatment of hepatic hydatid cysts (Utkan et al, 2001).

\section{Conclusion}

No doubt, hydatidosis due to Echinococcus granulosis is one of the most important zoonotic diseases not only in Yemen, but also worldwide. Owners of dogs must keep in mind that infected dogs are asymptomatic and human hydatidosis are typically asymptomatic except a few cases of long standing and heavy infections that may be fatal.

Omentoplasty gave none significantly decreased in total postoperative morbidity than partial cystectomy with drainage (PCD). Omentoplasty proved to be safe, simple, and effective and meet all criteria of surgical treatment of hydatid disease without intraoperative spillage and saving healthy tissues There was significant decreased in the mean postoperative hospital stay between the two groups. Omentoplasty is better than 
PCD as mean for surgical management of hydatid cysts of the liver

\section{References}

Abbas, M, Nafeh, AI, Youssef, YF, Nasr, MM, Radwan, HS, 2006: Conservative versus radical surgery for treatment of uncomplicated hep- atic hydatid cysts. J. Egypt. Soc. Parasitol. 36, 2:559-76.

Doty, JE, Tompkins, RK, 1989: Management of cystic disease of the liver. Surg. Clin. North Am. 64, 2: 285-95

El-Sebaie, SB, El-Sebae, MMA, Esmat, M E, Majid, M, Nasr, MM, Kamel, MM, 2006: Modified endo-cystectomy versus pericystectomy in Echinococcus granulosus liver cysts: a randomized controlled study, and the rule of specific anti-hydatid IgG4 in detection of early recurrence. J. Egypt. Soc. Parasitol. 36, 3:995-1008.

Eser, I, Karabag, H, Gunay, S, Seker, A, Cevik, M, et al, 2013: Surgical approach for patients with unusually located hydatid cyst. Ann. Ital. Chir. 2Nov 18;84. pii: S0003469 X13021337.

Fan, Y, Liu, Y, Lin, J, Chang, X, Wang, W, et al, 2013: Drainage does not promote postoperative rehabilitation after bilateral total knee arthroplasties compared with nondrainage. Chin. Med. Sci. J. 28, 4:206-10.

Grossi, G, Lastilla, MG, Teggi, A, et al, 1991: 420 patients with hydatid cyst: observations on the clinical picture. Arch. Hydatid. 30:1021-5.

Haridy, FM, Holw, SA, Hassan, AA, Mo-rsy, TA, 2008: Cystic hydatidosis: a zoonotic silent health problem. J. Egypt. Soc. Parasitol. 38, 2:635-44.

Hołody-Zaręba, J, Zaręba, KP, Kędrak, B, 2013: Assessment of the accuracy of preoperative imaging methods in the diagnosis of hepatic single-chamber echinococcosis. Pol. Przegl. Chir. 85, 12:693-8.

Hotez, PJ, Savioli, L, Fenwick, A, 2012: Neglected tropical diseases of the Middle East and North Africa: review of their prevalence, distribution, and opportunities for control. PLoS Negl. Trop. Dis. 6, 2:e1475. doi: 10.1371/journal.pntd.0001475.

Hussein, EM, Al-Mohammed, HI, AlMulhim, AS, Aboulmagd, E, 2012: HLA class II DRB1 resistance and susceptible markers in hydatidosis saudi patients in association to the clinical course and gender. J. Egypt. Soc. Parasitol. 42, 3:573-82.

Just, BA, Kern, P, Luthardt, RG, Rudolph, H, Grüner, B, et al, 2014: Echinococcus cysts affecting oromaxillofacial structures- a systematic review. Oral Dis. Feb 5. doi: 10.1111/odi.12225.

Kassem, HH, Abdel-Kader, AK, Nass, SA, 2013: Prevalence of hydatid cysts in slaughtered animals in Sirte, Libya. J. Egypt. Soc. Parasitol. 43, 1:33-40.

Khuroo, MS, Wani, NA, Javid, G, Khan, B A, Yattoo, GN, et al, 1997: Percutaneous drainage compared with surgery for hepatic hydatid cysts. N. Engl. J. Med. 337:881-7.

Kizildag, B, Dagistan, E, Gurel, S, Alan, C, 2013: CT and MRI findings of renal infestation by a huge active hydatid cyst. BMJ Case Rep. Jul 5;2013. pii: ber 2013010472

Ozkan, F, Yesilkaya, Y, Peker, O, Yuksel, M, 2013: Anaphylaxis due to spontaneous rupture of primary isolated splenic hydatid cyst. Int. J. Crit. Illn. Inj. Sci. 3, 2:152-4.

Palanivelu, C, Rangarajan, M, Senthilkumar, R, Madankumar, MV, 2007: Laparoscopic management of symptomatic multiple hepatic cysts: a combination of deroofing and radical excision. JSLS. 11, 4:466-9.

Ramia, JM, De-la-Plaza, R, Quiñónes, J, Adel, F, Ramiro, C, et al, 2013: Frank Intrabiliary Rupture in Liver Hydatidosis Located in the Hilar Plate: A Surgical Challenge. Dig. Surg. 30, 4/6:439-43.

Safioleas, MC, Misiakos, EP, Kouvaraki, M, Stamatakos, MK, Manti, CP, et al, 2006: Hydatid disease of the liver: a continuing surgical problem. Arch. Surg. 141, 11:1101-8.

Sahin, M, Koksal, H, Yilmaz, H, Cakir, M, 2010: Surgical treatment of hepatic hydatid cyst: cysto-jejunostomy by stapling. Bratisl. Lek. Listy 111, 6:349-50.

Tan, A, Yakut, M, Kaymakçioğlu, N, Ozerhan, IH, Cetiner, S, et al, 1998: The results of surgical treatment and percutaneous drainage of hepatic hydatid disease. Int. Surg. 83, 4:3146.

Traibi, A, El Hammoumi, M, El Oueriachi, F, Arsalane, A, Kabiri, EH, 2012: Benign cysts of the mediastinum: series of 28 cases. Rev. Mal. Respir. 29, 9:1111-5. Ghartimagar, D, Ghosh, A, Shrestha, M K, Talwar, OP, Sathian, B, 2013: 14 years hospital based study on clinical 
and morphological spectrum of hydatid disease. JNMA J. Nepal Med. Assoc. 52, 190:349-53.

Utkan, NZ, Cantürk, NZ, Gönüllü, N, Yildirir, C, Dülger, M, 2001: Surgical experience of hydatid disease of the liver: omentoplasty or capitonnage versus tube drainage. Hepatogastroenterol. 48, 37:203-7
Yucesoy, C, Ozturk, E, Hekimoglu, B, 2013:

Radiologic findings and percutaneous treatment of a rare giant soft tissue hydatid cyst. JBR-BTR 96, 5:286-9.

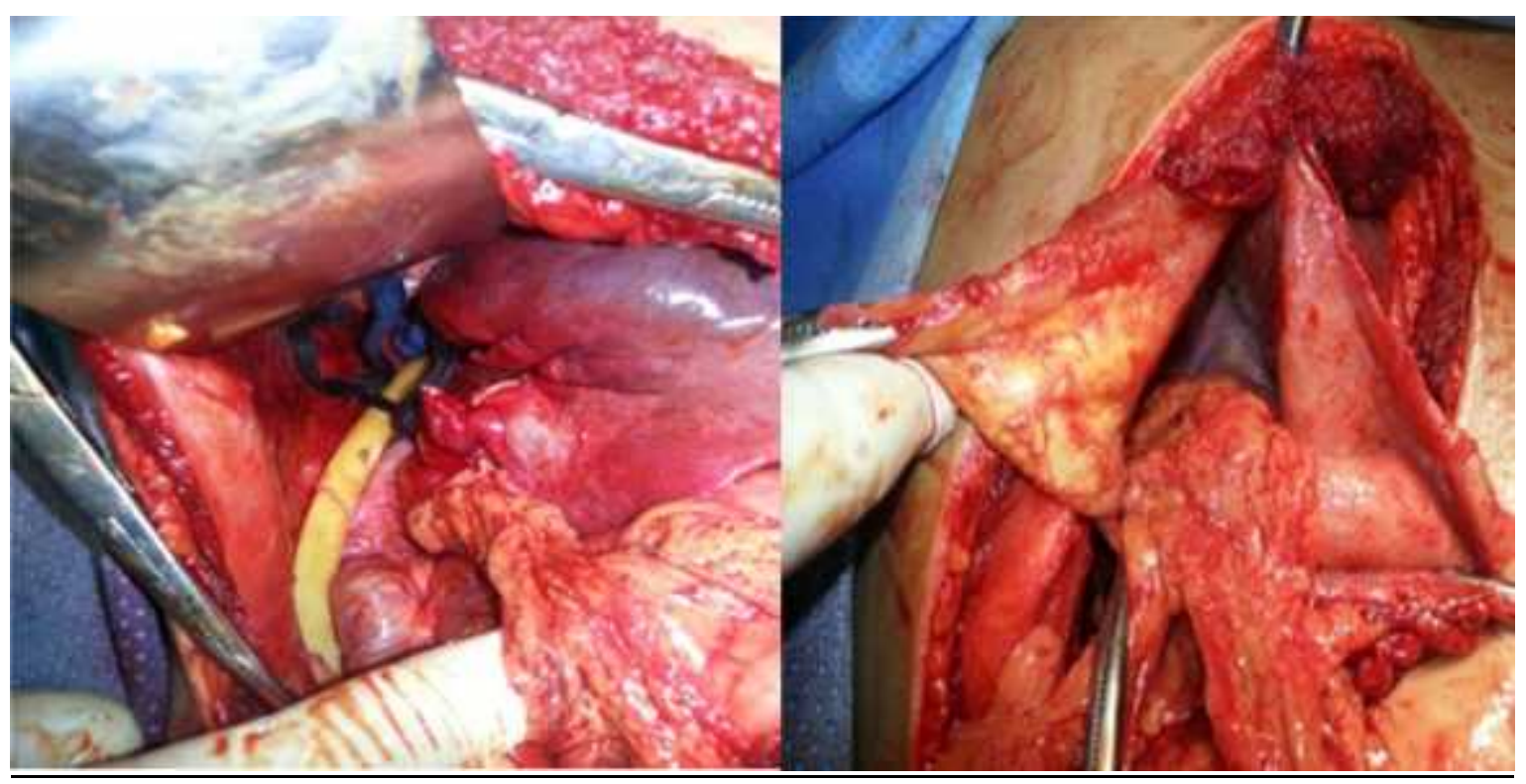

Fig. 1: Omentoplasty a-insertion of omentum b-suturing of edge
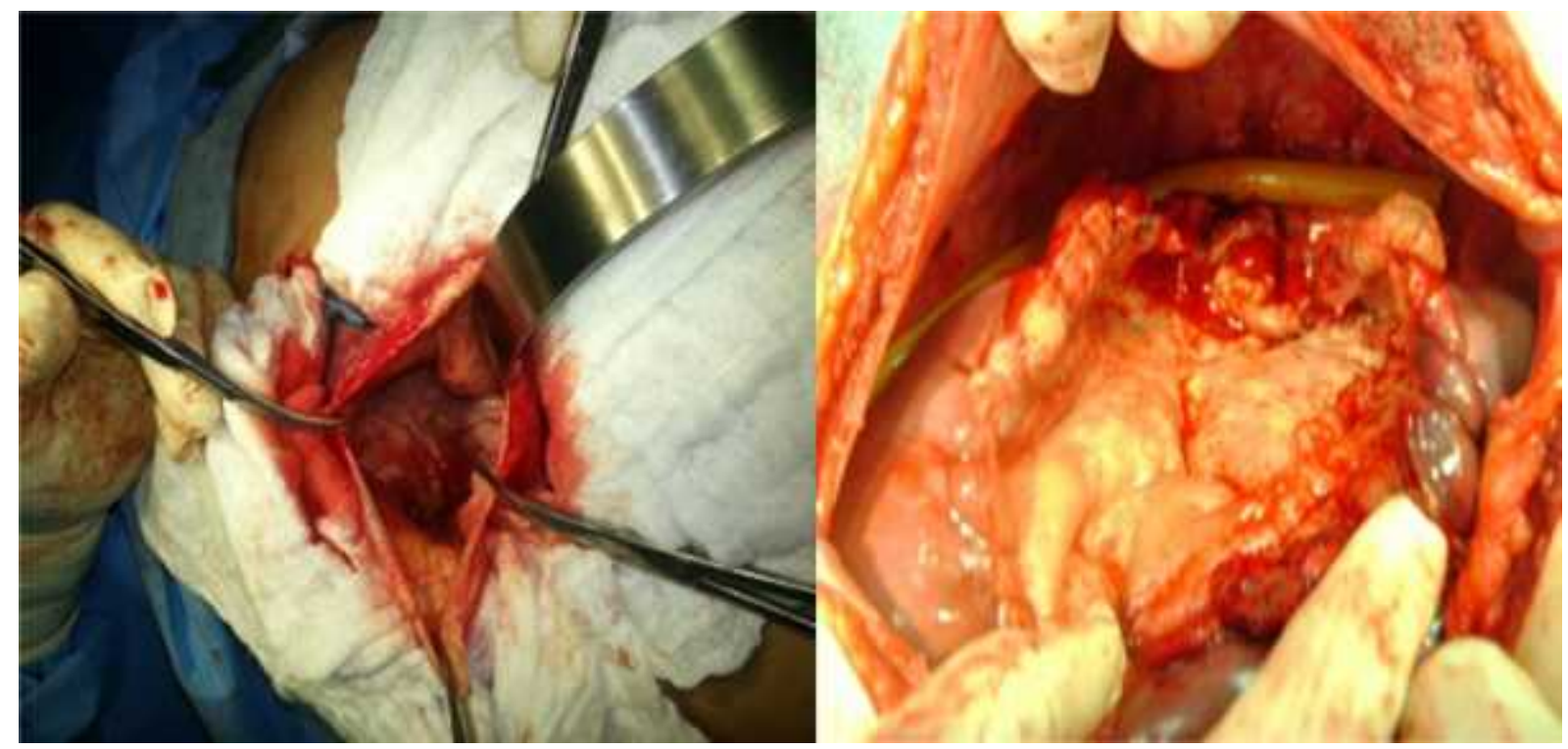

Fig. 2: PCD a-evacuation of cyst b-partial excision with drainage 